\title{
Lifecycle Problems in Consequence Estimation
}

\author{
William Lehman ${ }^{1, a}$ \\ ${ }^{1} 609$ Second St, Davis CA, 95691, United States of America
}

\begin{abstract}
The United States Army Corps of Engineers (USACE) guidance documents require economists and planners to meet a very high standard when evaluating consequences resulting from flood events. The guidance documents require analysts to evaluate how government action changes the consequences over time, in addition to evaluating how government inaction changes consequences over time as well. Corps guidance (Engineering Regulation 1105-2-100, Section 2-3.c.4 and Engineering Regulation 1105-2-100, Section 2-3.b respectively) require the evaluation of direct and indirect economic impacts, life risk impacts, and agricultural impacts for both current conditions and future most likely conditions across a range of alternatives. Evaluating the potential for impacts from flooding across time, and how time impacts their existence or vulnerability, is considered a "lifecycle approach". Little to no guidance is available on the process of calculating this "lifecycle approach" regarding changes in the value and number of assets within the floodplain over time. Performing a lifecycle analysis of a project over durations from 30 to 100 years, depending on the project purpose, requires evaluation of the changes in human behavior caused by changes in the floodplain such as reconstruction of structures, maintenance of structures, construction of new structures, population growth, and what type of structures are being built within the study area. This type of evaluation is not fully supported by most of the software programs utilized for flood risk management in the planning context. This paper is intended to describe pros and cons of economic lifecycle evaluation techniques to address the needs stated by policy, and tools that are being developed to support this analysis.
\end{abstract}

\section{Discussion of Scope}

Within the United States Army Corps of Engineers, there are many requirements defining how to evaluate flood risk within the context of flood risk mitigation. Minimal requirements are to investigate the with and without project conditions. The both conditions must contain some description of the flood risk a location experiences currently, and at some time in the future. These requirements are documented in ER 1105-2-100 [1], and more broadly documented in the Planning Principles and Guidelines of 1983[2]. The P\&G document of 1983 is applicable to any large scale infrastructure built by any federal agency within the United States for water related purposes.

Broadly speaking the metric for decision making is ultimately a benefit to cost ratio, which ensures that dollar for dollar the expenditure of the government is met or exceeded by the benefit of the infrastructure project. Of course, these benefit to cost ratios are constructed using a stream of forecasted benefits and cost along some hypothetical lifetime of the project, and brought back into consistent time dollars using discounting.

An additional alternative filtering mechanism is the reasonable maximization of net benefits ([2] Foreword 5.a) (benefits minus costs) which will ensure that the

\footnotetext{
${ }^{a}$ Corresponding author: William.p.lehman@usace.army.mil
}

federal government will receive the maximum benefit for each dollar spent. This filter is applied prior to the evaluation of a benefit to cost ratio, of course, any positive net benefit will create a benefit to cost ratio greater than unity.

To calculate a benefit and cost stream requires the evaluation of the current condition and the future condition with and without any governmental intervention. This is referred to as alternative analysis, but all alternatives must be compared to the no governmental action condition. This comparison yields benefits by subtracting the losses due to catastrophe in the alternative from the losses in the no action condition. Positive values indicate that the alternative in question reduced the losses due to catastrophe.

The cost to produce the reduction in losses is then calculated, and subtracted from the benefits to yield net benefits for the alternative in question. If the net benefit value is positive, it could potentially be a viable alternative. Other alternatives must go through the same process to create a range of viable alternatives, so that the analyst can maximize the net benefits to support plan selection and implementation.

Generally, all infrastructure investment strategies contain these basic principles, and require some analysis 
of now and the future with and without the intervention of the infrastructure project.

\section{Problem Statement}

Within the documents that govern how Federal agencies perform benefit to cost analysis, there are very many considerations that should be included within any analysis. Foremost is the requirement in section 2-3(b) of ER 1105-2-100[1] that the analyst "forecast critical resources relevant to the problems and opportunities in the floodplain... for selected years over the period of analysis". Interestingly, for USACE, future development cannot be claimed as part of the benefit stream if it is in locations that were previously defined as being in the 100 year floodplain unless structures are built above the 100 year floodplain elevation [3] . However, it is required that the analyst forecast future that construction regardless of first floor elevation to evaluate the impacts of hazard creep on the risk within the system, this is generally referred to as residual risk or induced risk [1] Chapter 3-3(2)(c) and 3-3(4).

To evaluate the without governmental action condition requires the analyst to forecast how people will behave (what structures they will build or abandon, how many children they will have, how often they will maintain their houses), how existing infrastructure will behave (will the existing mitigation structures fail, how often, when, where, will they be maintained, how often, in what ways), and how the loading of the system will change (will climate change impact the loading of this project, what about sea level rise, what about soil subsidence) without any governmental action. In addition to these complicated natural and human processes, there are many other less simple to evaluate impacts such as how upstream cottonwood recruitment will impact sedimentation within the system impacting loading through bed migration where the project is being evaluated.

How governmental action will change the system can be even more difficult to evaluate. If action is taken, will people build more houses, have more children, or continue as if nothing changed? Will the economy grow because new industrial parks are able to open, causing an influx of workers and capital into previously uninhabitable areas? When will this happen in the project lifecycle?

The transfer of risk from one location to another is expected to be considered (as an externality of the alternatives being considered), the effects of the project on the environment, the local economy, the national economy, and other societal impacts should be considered. The transformation of the hazard from slow steady rising flood to sudden catastrophic failure of defenses is to be considered as well.

The Hydrologic Engineering Center is a part of the Institute for Water Resources, and is responsible for assisting USACE in developing tools to meet the requirements of relevant guidance documents. HECFDA (Flood Damage Assessment) [4] was created to assist analysts in meeting the objectives stated by ER 1105-2-100 [1] and the Principles and Guidelines [2].

To simplify discussion, this paper will describe risk as a function of hazard, performance, exposure, and vulnerability. Within each component of the risk function, there are many life cycle issues. See Figure 1.

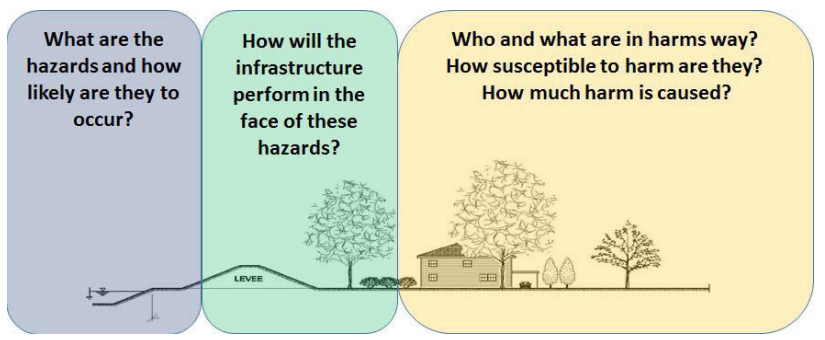

Figure 1 Components of Risk Function

HEC-FDA allows users to define four main relationships at locations within the watershed. The five main relationships are the flow frequency curve, the rating curve, the geotechnical performance curve, and the aggregated stage damage curve. The flow frequency curve and rating curve combine into a stage frequency curve, which generally describes the hazard component of risk. The geotechnical performance curve summarizes the performance component of risk. Finally, the aggregated stage damage curve summarizes both the exposure and vulnerability of damageable assets for a floodplain.

These relationships are combined to create a damage frequency curve. The damage frequency curve is then integrated to create Expected Annual Damages. This Expected Annual Damage estimate is used to identify benefits by comparing the with and without project conditions, and represents either the current or future year condition.

To assist in forecasting future conditions, HECFDA allows users to define a most likely future year, however, this definition is unrelated to the sequence of storms that occur within the lifetime of the project. This means that the analyst must describe the inventory without knowing what storms may have occurred. In the United States, New Orleans is only recently returning to a normal real estate market. Structures destroyed by Hurricane Katrina have still not been rebuilt, and most construction in the past ten years has been focused on rehabilitation and reconstruction from damage due to Hurricane Katrina. Evaluating the impact of large catastrophic events on the subsequent inventory is incredibly difficult to describe within the HEC-FDA architecture.

The relationships described above are defined at index locations within HEC-FDA, and each index location is represented as being statistically independent of one another. While it is possible to evaluate the 
conditional nature of upstream failures on downstream loading, it is incredibly difficult to adequately describe the system component necessary for the evaluation of risk transfer or risk transformation.

Given the policies that govern USACE, and the importance of water resources problems as population is continuing to increase, are there better approaches to evaluating risk within a watershed that recognizes the complexity of physical systems and human behavior across time? The next few sections will delve deeper into the complexities for each component of risk, and then discussion of a different modeling framework will explain how these problems could be modeled. Finally, benefits and limitations will be discussed and conclusions will be drawn.

\section{Hazard}

The hazard is the thing that is causing harm or causing the catastrophe. For USACE the hazard is typically flood risk from rivers or from storm surges on the coast.

A vast majority of large scale infrastructure projects seek to mitigate risk through manipulation of the hazard. These structural measures create defenses that keep the hazard away from assets and individuals that would be damaged by the hazard. Examples include levees, dams, and diversions, these projects seek to decrease the severity of the event in some locations, by moving the hazard elsewhere. Inevitably this causes other locations to have higher loadings from the same event. Typically the system is designed so that the places where the hazard is transferred to are designed to accommodate that increased loading, or in some cases actually benefit from the increased loading.

There are many forces that change loading outside of the actions of the federal government, and those actions will need to be evaluated in the without project condition. Examples include climate change, sea level rise, subsidence, scour and deposition of sediment. While some of these may be impacted by government action, many will continue to change the nature of the hazard regardless of governmental action.

Generally engineers are exceptionally robust at evaluating the current with and without conditions for hazard, but there are less engineers focused on evaluating the future, and how intervention will change the withproject future condition.

\section{Performance}

Performance is how the system and its components respond and perform to the loading. The way that performance of large infrastructure projects is characterized is usually a combination of hydraulic, structural, and geotechnical engineering.
The performance of infrastructure is not simply a fail or no fail type of analysis, but rather a continuum of failure modes, progression rates, and failure states. If the failure mode is levee breach, the duration of time for the breach to form and reach final breach width has tremendous impact on the inflow hydrograph, which can dramatically change the timing of the event. This can have very large impacts on the overall performance of the system.

Overtopping and breaching of levees higher in the system may decrease loading on downstream features substantially by changing the shape of the loading hydrograph. This impact can substantially change the overall consequences of an event all things being equal.

Evaluating the performance of a system, requires some level of evaluation of the performance of its components. To fully state the system response, the combinatorial component responses must be reasonably traversed. This is true for the without government intervention, and the with government intervention analysis.

The performance of components of a system are generally described using probabilistic definitions such as fragility curves. Evaluating fragility curves is very difficult to do, and many issues may arise. Within levees there are many issues that can make defining the response of existing infrastructure difficult to describe. Examples include the length effect, considerable knowledge uncertainty of what quality the levee construction materials and methods were utilized, and if there are existing issues that have not yet yielded visual cues externally to the levee.

Small changes to the system can dramatically change the overall response the system provides across time, by changing the nature of the system use or response, different maintenance problems can arise within the system.

\section{Exposure}

Exposure describes the presence of damageable assets or individuals within the area impacted by the hazard. In traditional benefit to cost analysis for flood risk management, structures and their contents expressed in dollar value is the primary category of exposure.

The evaluation of life loss has increased exposure to consider the people in the area impacted by the hazard. There are other categories that may be considered under exposure, but this paper will not endeavor to address all categories of exposure.

Evaluating exposure in the current condition presents some minor hurdles, but essentially the methods for describing structure values, content values, and population within a hazard area are already well defined. 
Describing exposure across time becomes a bit more difficult. Approximating the growth (in terms of structures, their values, and the population) can be accomplished using forecasted datasets that are publically available. This is only a part of the picture. In the without project condition, structures will be exposed to the hazard as they are with natural changes to the hazard and system performance. Some structures will be damaged, and evaluating if these structures will be rebuilt is critical for the evaluation of the future without governmental intervention condition. If individuals die due to flooding they will not be available to create population growth in the future. And for large events like hurricane Katrina, large scale migrations can occur.

Looking at changes to the future due to the withgovernmental intervention future condition, requires analysis to determine if the changes in the without governmental intervention future would still occur. But this is only part of the issue, if the hazard is mitigated, will this induce migration or development within the previously impacted regions? To what scale? Empirical precedence for population growth behind large scale infrastructure abounds, in California population behind the levees in Natomas caused growth of population from 60,000 to 120,000 from 1990 to 2000 .

\section{Vulnerability}

Vulnerability describes how much damage will occur to an asset as a function of the severity of the hazard. For most damage estimates to structures a driving parameter is water depth. Another critical parameter is the construction type and quality within the hazard area. If structures are prone to frequent flooding, the construction practices of structures in that location may be very different if the likelihood of flooding is low. If governmental intervention occurs, it is possible that less costly construction practices may be chosen thus increasing the vulnerability of the future structures.

As population ages, they become more vulnerable to hazards. In both the with and without governmental intervention future conditions, the age demographic of the exposed population can have a tremendous impact on the vulnerability of the population in an area effected by a hazard.

\section{Risk Assessment Using System/Lifecycle Approach}

It is critical that the components above be measurable, in that they must be able to be quantified consistently for the with and without the intervention of the government alternatives. There are many tools that assist the analyst in doing this quantification.

Tools have long been used to assist individuals in decision making. Primitively, numbers were constructed as a tool to help quantify things so that decisions can be made. Currently, new software programs are being released that can compute billions of computations at the push of a button, with increasing precision. The goal of these tools is to represent the physical reality of a system so that hypothetical changes can be evaluated without their physical manifestation. Unfortunately, a tool is only as good as the materials it is working with, the materials it is made from, and the user of the tool. Any fault in any of these elements will lead to outputs that are intrinsically flawed.

When considering the use of tools it is important to understand the intent of the tool, utilizing a tool for an unintended purpose can have disastrous results. However, learning to properly use and maintain the simple tools can yield very useful and meaningful insights into many issues.

Understanding the problem in question, and what factors impact the problem is essential when considering tool selection. Properly stating the problem and defining the framework for alternative selection can guide a multi discipline team to make better modeling decisions. These decisions can lead to more fruitful analysis and comparison of the alternatives in question.

The systems modeled are intrinsically complex, and require a range of analysis techniques, data sources, computational methods, and interpretations. Representing the physical system with the appropriate accuracy and precision is difficult, but it can be done. Understanding that precision does not imply accuracy is critical to choosing the proper techniques and data sources. Knowing when the modeled system is as simply stated as possible without being any simpler than necessary is difficult, and requires firm understanding of the decisions being made from the information being provided.

HEC-FDA [4] uses general relationships at index locations to describe a system, while this is useful in evaluating the system at that location, some of the system components are lost in this analysis. To include the intricacies of the system it is necessary to represent the system in a more robust way. For clarification, Figure 2 shows a general schematic of a system.

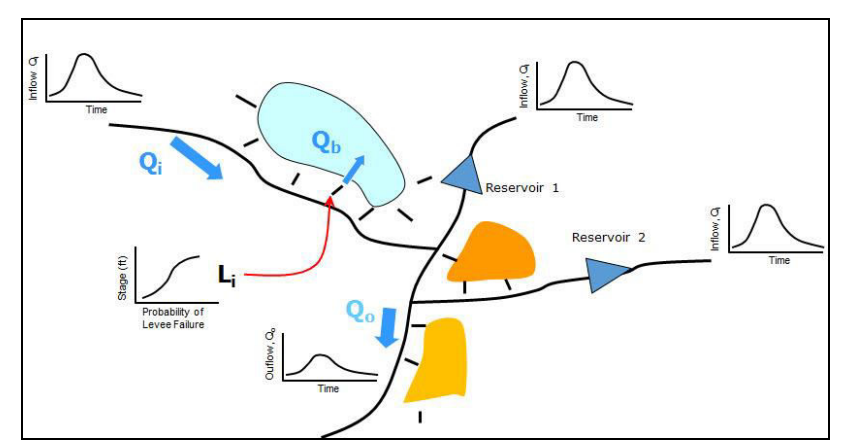

Figure 2 General schematic of a System

Within the system there are many components, reservoirs, levees, diversions, or channel modifications. Each of these components behave differently, and their behavior is defined in part by the nature of the event 
being modeled. If there is a breach at location $\mathrm{L}_{\mathrm{i}}$ the flow $\mathrm{Q}_{\mathrm{b}}$ will be removed from the system, attenuating the flow as it moves down stream. This reduction in flow will possibly result in a lower flow downstream and thus a lower stage on any subsequent levee system. The attenuation will also possibly increase the duration of flow at a downstream levee, and this increased duration may cause higher likelihood of failure. Within a system, changes during an event can have dramatic unanticipated changes to the performance of the system as a whole.

Many of the tools developed for hydraulic or hydrologic analysis can model these subtle issues. A drawback to the detail is that running these high fidelity models can be computationally prohibitive. Or that the models themselves can only evaluate one event at a time.

HEC-WAT (Watershed Analysis Tool) [5] has been developed to assist analysts to describe a system with single event models such as HEC-HMS [6], HEC-ResSim [7], HEC-RAS [8], and HEC-FIA [9]. This enables the systems approach for risk assessment. HEC-WAT generates storms based on the analysts input hydrology, and runs the single event models in sequence for each event generated. This allows many events to be aggregated together to span the frequency range, and ultimately to combine the Hazard, Performance, Exposure, and Vulnerability of the system into an expression of Risk using Expected Annual damages.

Similar to HEC-WAT, HR Wallingford has created the RASP tool which enables a systems based approach to evaluating flood risk for a watershed.

While both of these tools do an excellent job at evaluating the systems approach, neither describe the way the system changes across time very well. HEC-WAT is being designed to assist in lifecycle analysis [5], but technically it is not yet meeting the objective completely. To do this each event must be computed in a sequence of years that represent the analysis period for the project, and the state of the system needs to be preserved after each event. If a levee breaks, the levee would need to remain broken until it is repaired, and after the repairs, the levee performance would need to reflect the repairs for any subsequent event. These types of state variables would need to be preserved for all components of the risk equation.

Currently the tools available do not provide this type of assistance to the analyst. Typically the assumptions within the tools available is that the state of the system goes back to its original state immediately after the event. This assumption can lead to overstating or understating risk. By instantaneously rebuilding structures, more damageable value is available in the flood plain than is reasonable to expect thus overstating risk to some degree, but by not tracking the degradation of levees across time risk will be understated.

\section{Alternative Analysis}

While the goal of these tools is to produce truth, it is rarely achieved. However, the decisions made through the aide of these tools can be unhindered by the imprecision or inaccuracies of the tools themselves. Since our metric is the comparison of damages with and without a project, if the same tool is used for the with and without project, the same intrinsic bias should be present in both outputs. This should lead to relative differences that are meaningful to decision makers.

Alternatives can be compared in many ways, however, some methods require different analysis techniques. If alternatives are being compared to absolute thresholds or guidelines, such as the Tolerable Risk Guidelines, the alternatives must be stated in terms of absolute risk. This means that bias within the modeling framework can dramatically change the decisions being made. If alternatives are being compared using benefits the evaluation is intrinsically a relative comparison which can reduce the impacts of bias (provided the bias is consistent and linear within the modeling framework). However, to know that the cost of one alternative is significantly larger than the cost of another alternative which bears the same benefit, the lower cost alternative should be selected. Introducing cost into a benefit to cost ratio or net benefits calculation requires the analyst to use the same level of detail in evaluating the cost as the benefit. If care is taken, and the costs are defined in a similar and unbiased way across all alternatives, the measure of benefit to cost ratios and net benefits are still relative. Developing alternative comparisons in a relative way allows the analyst to use methods to approximate certain relationships, thus alleviating the quest for accuracy.

While the last paragraph may seem an argument to reduce the complexity of the modeling utilized, it is not necessarily intended to be such an argument. Our systems are innately complex, but our evaluation of them can use simplifying assumptions. Accurately depicting truth is noble, but not always necessary. Doing sufficient evaluation to determine if certain relationships are important is necessary, and sometimes this requires very complex evaluations. Before we can make simplifying assumptions, it is important to determine if the simplifying assumption will impact the decision being made.

\section{Decision Making}

The process of modeling the risk of a system within a lifecycle is by nature done to inform some level of decision. Any element added to the framework that models the hazard, performance, exposure, and vulnerability of a system should be carefully considered. The consideration should take into account how critical the element is to defining the risk of the system, the comparison of alternatives, and defining how things change across time. If any element has no impact on the decision being made, it should not be modeled because it 
then becomes a source of error within the system that is not necessary.

\section{Benefits and Limitations}

Evaluating risk within a lifecycle approach can dramatically change the solutions considered within a risk informed decision making process. It requires the analyst to evaluate how actions change the future. This is innately a good thing. However, adding complexity to a process that is beyond the capability of most tools is very difficult to swallow as an analyst and as a decision maker.

\section{Concluding Remarks}

The framework stated by Principles and Guidelines written in 1983 [2] requires analysis that seems very difficult to evaluate with the tools that exist today. Analysts tend to study risk in perpetuity without making any relevant finding of how to effectively manage risk. Part of the reason may be due to developing models and analysis that is not directly in support of decision making, and providing detail well beyond that necessary to describe the risk of a system. Additionally, so much time is spent evaluating detail, that the system is not evaluated sufficiently or at all, thus making it very difficult to provide the information decision makers need to sufficiently address the risk of the system.

\section{References}

1. USACE, 2000. Planning Guidance Notebook, Engineering Regulation 1105-2-100. USACE, Washington D.C.

2. 1983. Economic And Environmental Principles And Guidelines For Water And Related Land Resources Implementation Studies

3. Executive Order 11988, 1997, Flood Plain Management

4. Hydrologic Engineering Center, 2014. HEC-FDA, Flood Damage Analysis User's Manual, Version 1.4, CPD-72, U.S. Army Corps of Engineers, Davis, CA.

5. Hydrologic Engineering Center, 2016. Flood risk management with HEC-WAT and the FRA compute option, FLOODrisk2016.

6. Hydrologic Engineering Center, 2015. HEC-HMS, Hydrologic Modeling System User's Manual, Version 4.1, CPD-74A, U.S. Army Corps of Engineers, Davis, CA.

7. Hydrologic Engineering Center, 2013. HEC-ResSim, Reservoir System Simulation User's Manual, Version 3.1,CPD-82, U.S. Army Corps of Engineers, Davis, CA.

8. Hydrologic Engineering Center, 2016. HEC-RAS, River Analysis System User's Manual, Version 5.0, $C P D-68$, U.S. Army Corps of Engineers, Davis, CA.

9. Hydrologic Engineering Center, 2016. HEC-FIA, Flood Impact Analysis User's Manual, Version 3.0, CPD-81, U.S. Army Corps of Engineers, Davis, CA. 